\title{
ANALYSIS OF HUMAN PERCEPTION ABILITY FOR ROBOT IMPEDANCE
}

\author{
Yoshiyuki Tanaka ${ }^{* 1}$ Toshio Tsuji ${ }^{*, 1}$ \\ Hideki Miyaguchi ${ }^{* *}$ \\ * Graduate School of Engineering, Hiroshima University, \\ Higashi-hiroshima, JAPAN \\ ** Graduate School of Health Sciences, Hiroshima \\ University, Hiroshima, JAPAN
}

\begin{abstract}
This paper examines human impedance perception ability during manipulation of an impedance-controlled robot, especially as regards perception accuracy and differential limen. In the experiments, a subject is instructed to move the handle of an impedance-controlled robot and to perceive the presented value of the robot impedance. Experimental results show that both motor control ability and somatosensory information play important roles in impedance perception. Copyright (C)2005 IFAC
\end{abstract}

Keywords: Mechanical impedance, human impedance perception, sensory-motor integration

\section{INTRODUCTION}

Humans have highly developed sensory receptors and functions that allow us to survive in the environment. The sensory system enables us to receive and recognize various kinds of external information, ultimately leading to actions. Paradoxically, it becomes difficult or almost impossible for a patient with a sensory or perceptual disorder, whose sensory system does not function properly, to gain sufficient information about his environment. Many rehabilitation methods using motion exercises have been proposed for training damaged senses and perception, building on the well-known evidence that motion is an important influence on the abilities of the senses and of perception (Oyama et al., 1994). This is because perceptual ability is based on sensory-motor abil-

\footnotetext{
1 This research work was supported in part by a Grant-inAid for Scientific Research from the Japanese Ministry of Education, Science and Culture (15360226 and 16760203).
}

ity, which arises through the integration of motion and senses (Kephart, 1960).

A human regulates the dynamic properties of his musculoskeletal system to achieve target tasks. These dynamic properties of humans can be expressed by the mechanical impedance parameters (stiffness, viscosity, inertia), and a human can control his impedance properties by changing the configuration of his arm (Mussa-Ivaldi et al., 1985; Dolan et al., 1993; Tsuji et al., 1995). It is reasonable to suppose that a training method aimed at improving the ability to control human impedance properties would be very useful in sports and in rehabilitation. Previously, Tsuji et al. proposed the concept of an "impedance training method" to improve human impedance regulation ability (Tsuji et al., 1999). However, their method does not include impedance perception ability, although a close relationship exists between movement ability and the senses and perception, as pointed out above. 
Recently, a cognitive therapeutic exercise based on neurophysiology and learning theory has been put forward as a new approach to therapeutic exercise (Perfetti et al., 1998). Functional rehabilitation is achieved by using motion exercises associated with perception. A spring, a sponge, and a weight are used, with a trainee being asked to recognize and perceive the hardness and softness of these objects; that is, the impedance properties of the objects. However, it is not possible to present all of the relevant impedance properties in the cognitive therapeutic exercises using easily obtained objects. Moreover, an effective design method for a training program that takes into account the level of a patient's disorder has not been established, nor has a quantitative evaluation method for training effects been put forward. If a robotic device could be developed to present various impedances to a trainee easily, it would be well worth establishing a cognitive therapeutic exercise. For this, it is necessary to investigate human impedance perception ability and to clarify the important functions associated with impedance perception.

There have been several studies on human perception of viscoelastic properties. For example, Jones and Hunter reported that a human can perceive changes in stiffness and viscosity (Jones and Hunter, 1990,1993). In their experiment, a subject was asked to match the stiffness/viscosity of the force on his left hand to the perceived stiffness/viscosity of another force on his right hand. Srinivasan and LaMotte conducted experiments exploring the human ability to distinguish an object's hardness (Srinivasan and LaMotte, 1995), in which subjects were asked to press on an object with the tip of a finger and to recognize its stiffness using only the deep somatic sensation, without the presentation of the tactile sense. Then, they argued for the importance of the deep somatic sensation and the tactile sense. Fujita et al. discussed the contributions of the deep somatic sensation as well as of tactile and visual information to the stiffness perception of an object in finger grips, using the discrimination ratio of the perceived to real stiffness value (Fujita et al., 1999). However, none of these earlier studies carries out a quantitative analysis of perception ability with regard to impedance values. In addition, the mechanical factors that influence the human impedance perception ability have not been investigated.

The present paper examines human perception of robot impedance parameters, as the first step toward developing a training method based on cognitive therapeutic exercise for improving impedance perception ability. The results of this study will be useful for constructing novel rehabilitation systems. The study also provides basic data for use in determining robot impedance

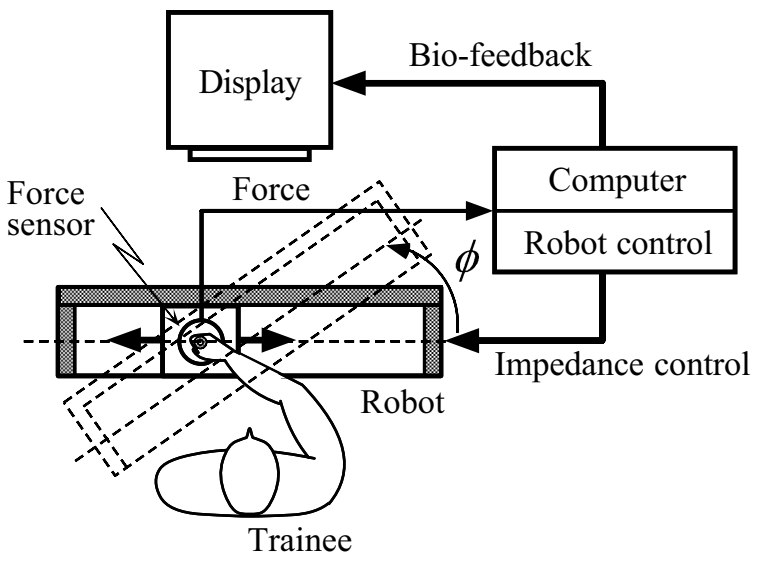

Fig. 1. Experimental Apparatus.
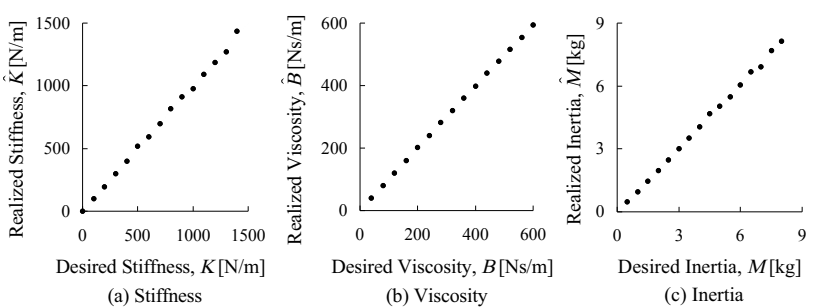

Fig. 2. Accuracy of impedance control used in the experiments. Mean values for 10 sets of the realized impedance are shown.

properties. This will assist the human operators in power-assist or human-robot systems (Kazerooni, 1990).

This paper is organized as follows. Section 2 explains the experimental apparatus and method for the impedance-perception experiment, and presents a series of experimental results. In Section 3 and 4, preliminary comparison experiments are carried out with normal healthy subjects. The importance of the motor functions and sensorymotor integration in impedance perception is then analyzed from the viewpoint of the mechanical power involved in human movements.

\section{EXPERIMENTAL METHOD}

\subsection{Experimental Apparatus}

Figure 1 shows an overview of the experimental apparatus, which includes a linear motor table with one degree of freedom (Nihon Thomson Co., Ltd., encoder resolution: $2[\mu \mathrm{m}])$, used to present impedance characteristics to subjects, a computer for robot control, and a display that shows training information such as position and hand force in the training. A handle and a six-axis force/torque sensor (BL Autotec Co., Ltd., resolution ability: 

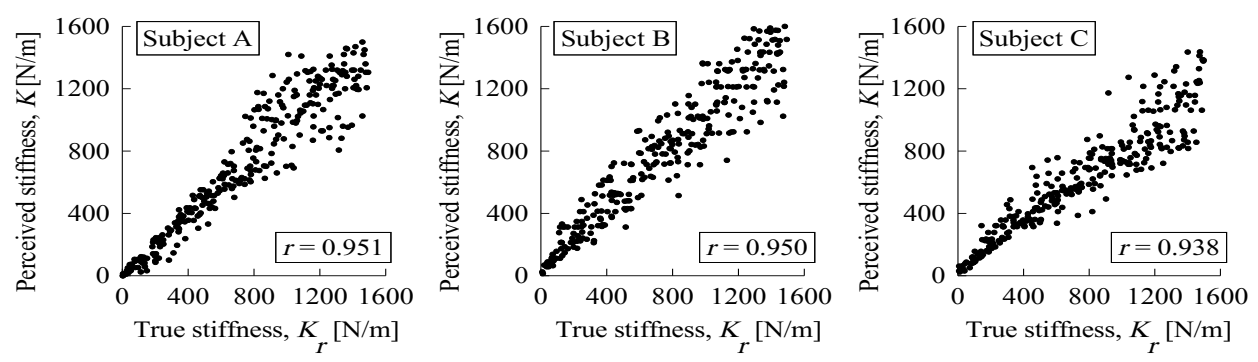

(a) Stiffness
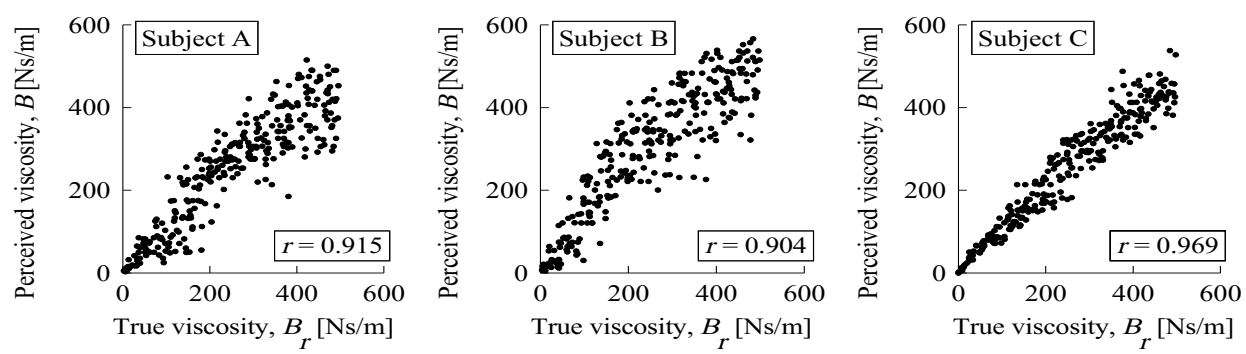

(b) Viscosity
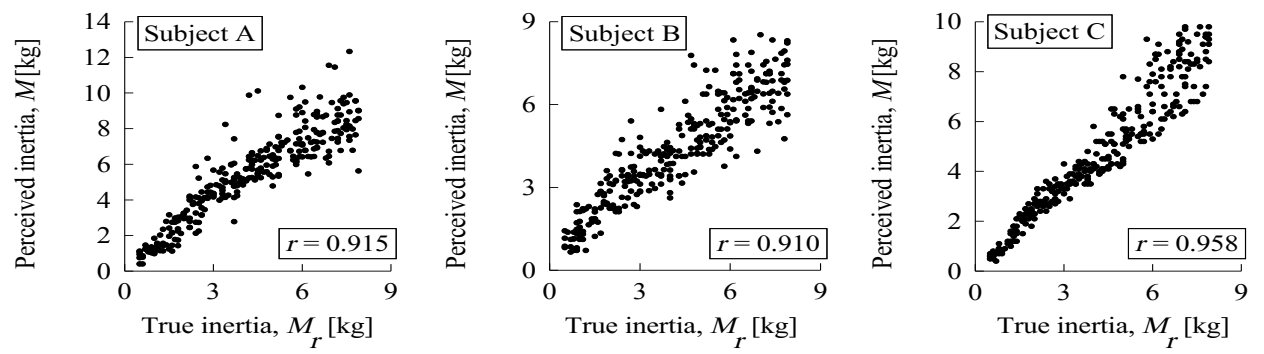

(c) Inertia

Fig. 3. Relation between true and perceived impedance values under Condition 1 by Subs. A, B and C.

force $x$ axis, $y$ axis: $0.005[\mathrm{~N}], z$ axis: $0.15[\mathrm{~N}]$, torque: $0.003[\mathrm{Nm}]$ ) are attached to the moving part of the robot to measure the operating hand force imposed by a subject. The handle (hand) position is measured by an encoder built into the linear motor table. The operational direction $\phi$ is changed by means of the rotary motor set under the table.

The robot in this system operates under the impedance control method (Hogan, 1985), so the dynamic behavior of the robot $R(\mathrm{~s})$ is given by

$$
R(s)=\frac{1}{M_{r} s^{2}+B_{r} s+K_{r}} .
$$

Figure 2 illustrates the accuracy of the robot impedance control described in this paper with respect to stiffness, viscosity and inertia. The vertical axis shows the actual impedance, while the horizontal axis shows the desired impedance. The actual impedance was estimated using the least squares method based on the measured data for position, velocity, acceleration and force over a 30second period when the subject handles the robot (Tsuji et al., 1999). Mean values for 10 sets of estimated results are plotted. The standard devi- ation for each trial is less than $0.05 \%$. It can be seen that all the elements of the robot impedance can be realized accurately and simultaneously.

\subsection{Experimental Procedure}

In the experiment, a subject is instructed to perceive the value of the robot impedance by moving the handle attached at the impedancecontrolled robot freely. The following three kinds of experiments are designed as follows.

1. The impedance perception ability was analyzed by changing one of the robot impedance parameters; stiffness $K_{r}$, viscosity $B_{r}$, or inertia $M_{r}$.

2. The impedance perception ability was analyzed by changing all robot impedance parameters simultaneously.

3. The difference limen for each of the robot impedance parameters was analyzed by presenting two different impedances in order. The subject was to judge which of the impedance values was bigger. 
The presenting order of the robot impedances was randomly determined for all conditions. Before the experiments, the simple preliminary training was performed in which a subject was asked to spend five minutes memorizing the feelings for some standard values of robot impedance. The robot impedance values represented four levels of stiffness $\left(K_{r}=0,500,1000,1500[\mathrm{~N} / \mathrm{m}]\right)$, six levels of viscosity $\left(B_{r}=0,100,200,300,400,500\right.$ $[\mathrm{Ns} / \mathrm{m}])$, and five levels of inertia $\left(M_{r}=0.5,2.0\right.$, $4.0,6.0,8.0[\mathrm{~kg}])$.

The perceived values of robot impedance were not revealed to subjects in these experiments. The experiments were carried out with six healthy subjects (male university students, aged $22 \sim 24$ ), following sufficient practice.

\section{HUMAN PERCEPTION ABILITY OF ROBOT IMPEDANCE}

Figure 3 illustrates the experimental results under Condition 1 for three subjects. The vertical (horizontal) axis indicates the perceived (true robot) impedance, and $r$ is the correlation coefficient between the true and perceived impedance values. The ranges of the true impedance were $0 \sim 1500$ $[\mathrm{N} / \mathrm{m}]$ for stiffness, $0 \sim 500[\mathrm{Ns} / \mathrm{m}]$ for viscosity, and $0.5 \sim 8.0[\mathrm{~kg}]$ for inertia. The resolution of the true impedances was set as $1[\mathrm{~N} / \mathrm{m}]$ for stiffness, $0.1[\mathrm{Ns} / \mathrm{m}]$ for viscosity, and $0.1[\mathrm{~kg}]$ for inertia. The range of each impedance parameter was divided into 15 sections. In all, 300 impedance values were identified, with 20 values in each section. The viscosity was set to $0[\mathrm{Ns} / \mathrm{m}]$ and the inertia to $2[\mathrm{~kg}]$ in the stiffness perception experiment; the stiffness was set to $0[\mathrm{~N} / \mathrm{m}]$ and the inertia to $2[\mathrm{~kg}]$ in the viscosity perception experiment; and the stiffness was set to $0[\mathrm{~N} / \mathrm{m}]$ and the viscosity to $0[\mathrm{Ns} / \mathrm{m}]$ in the inertia perception experiment. The results demonstrate that the subjects can perceive the presented robot impedance with considerable accuracy, since the correlation coefficient exceeds 0.90 , although the variance of the perceived impedance did increase as the true impedance increased. Similar tendencies were observed for the other three subjects.

The influences of arm posture and motion direction on the accuracy of impedance perception were examined by changing the operational direction of the robot $\phi=0,45,90$, and 135 [deg.], so that the shoulder of each subject was belted to the chair while the upper arm was almost on a horizontal plane. Other experimental conditions were the same as shown in Fig. 3.

Figure 4 presents one of our experimental results in the stiffness perception by Subject $\mathrm{C}$ with respect to the motion direction $\phi$. The range of
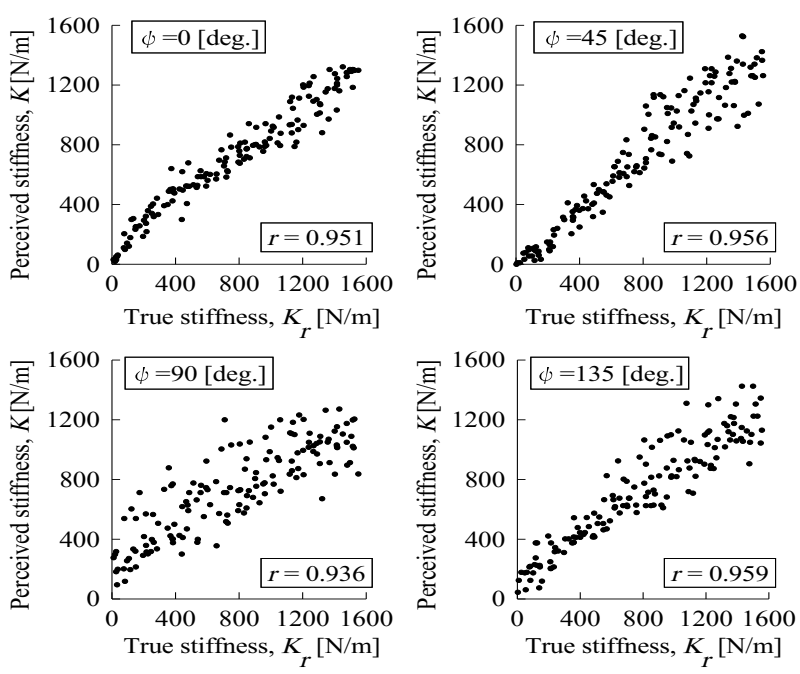

Fig. 4. Changes of the stiffness perception ability according to the motion direction

robot stiffness was divided into 15 sections, such that 150 values of stiffness were randomly presented in each section. The subject perceived the robot impedance with considerable accuracy even in the constrained posture, so motion direction does not significantly affect the impedance perception accuracy. The similar tendency was observed with the other two subjects.

The experiments involving Condition 2 were carried out with a specific combination of robot impedance parameters $(3 \times 3 \times 3=27$ trials $): K_{r}$ $=0,250,500[\mathrm{~N} / \mathrm{m}] ; B_{r}=0,50,100[\mathrm{Ns} / \mathrm{m}] ;$ and $M_{r}=0.5,1.5,3.0[\mathrm{~kg}]$. The combinations of robot impedance were randomly presented in each trial. Each subject was asked to respond to all three unknown impedance values.

Figure 5 shows the results for all subjects, with the vertical axis of each graph representing the correlation coefficient between the true and perceived impedance values. Perception accuracy drastically decreased compared with the results under Condition 1 (See Fig. 3). In addition, the perception accuracies for viscosity and inertia are significantly lower. This indicates that multipleimpedance perception was more difficult than single-impedance perception.

\section{DIFFERENTIAL LIMEN OF ROBOT IMPEDANCE}

The differential limen for the impedance parameters was investigated by using a constant method (JUSE the sensory test committee, 1973), in which a set of stimuli was presented to a subject in random order. The largest stimulus, causing a $95 \%$ reaction, was selected from a set of standard stimuli $S$ composed of all stimuli representing ten levels of stiffness $\left(K_{r}=100,200, \cdots, 1000[\mathrm{~N} / \mathrm{m}]\right)$, 

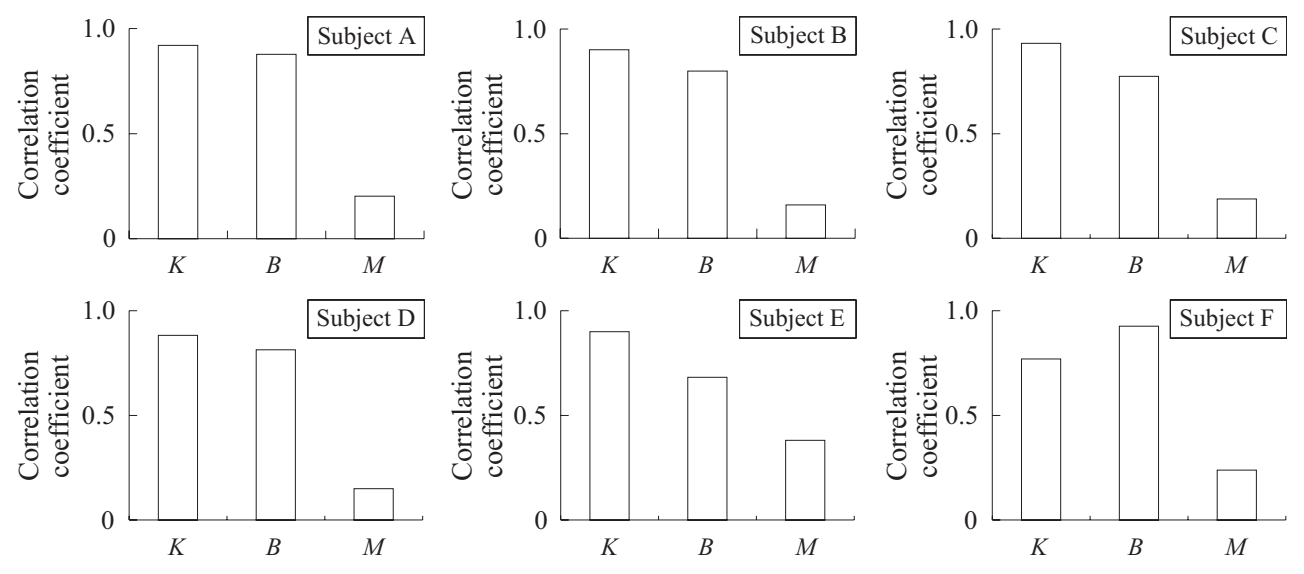

$K$ : Stiffeness, $B:$ Viscosity, $M$ : Inertia

Fig. 5. The correlation coefficients between true and perceived impedance computed from the experimental results where all of impedance parameters $K_{r}, B_{r}, M_{r}$ were perceived by the subjects simultaneously

eight levels of viscosity $\left(B_{r}=20,40, \cdots, 160\right.$ $[\mathrm{Ns} / \mathrm{m}])$, and eight levels of inertia $\left(M_{r}=1,2\right.$, $\cdots, 8[\mathrm{~kg}])$. A stimulation series was composed of $4 \sim 7$ different stimuli, where each stimulus is calculated by decreasing the largest value by regular intervals of $10[\mathrm{~N} / \mathrm{m}]$ for stiffness, $1[\mathrm{Ns} / \mathrm{m}]$ for viscosity, and $0.1[\mathrm{~kg}]$ for inertia. A second comparison stimulus was then randomly selected from the stimulation series. The subject was asked to compare the comparison stimulus to the standard stimulus, and to describe his result as "It is smaller," "It is the same," or "It is bigger." The experiment was repeated 20 times, changing the presentation order of the comparison stimuli (Shiomi et al., 1982). The number of correct answers in each stimulation series was analyzed using a $\mathrm{T}$ test (a two-tailed test of $1 \%$ level of significance) with a binomial distribution. The differential limen $\Delta S$ was defined as the smallest difference between stimuli that a subject could discriminate.

Figure 6 illustrates the discrimination ability for all impedance parameters for Subjects A, B, and C. The vertical and the horizontal axes show the differential limen and the standard stimulus impedance. The dotted line is the regression line, calculated by the least-squares method. The equation for the regression line is also presented in the figure. The differential limen increases as the standard stimulus impedance increases. A similar tendency was observed for the other three subjects. This tendency corresponds to the observations of impedance perception ability discussed in Section 3. It should be noted that the relationship between the standard stimulus value, $S$, and the differential limen of the impedance, $\Delta S$, is almost proportional. That is, Weber's law (JUSE the sensory test committee, 1973) is almost satisfied in the differential limen of robot impedance.

\section{CONCLUSION}

This paper experimentally analyzed human impedance perception ability, especially as regards perception accuracy and differential limen. The experimental results revealed the following points.

- When the true impedance is small, the differential limen is small, and the perception accuracy is considerably high.

- Multiple-impedance perception is more difficult than single-impedance perception. In particular, perception accuracy for viscosity and inertia is remarkably lower.

- The differential limen increases proportionally to the true impedance. The value of perceived impedance disperses when the differential limen is large.

- Human impedance perception almost follows Weber's law.

In the future, it will be necessary to examine in detail the cause of the reduction in accuracy during multiple-impedance perception in detail, especially inertia. Finally, we plan to develop an impedance-perception training system based on cognitive motor therapy. This will involve setting up clear training indexes and objectives, as well as effective rehabilitation of tactile cognition and discrimination in cases of sensory and perceptual dysfunction, particularly tactile perceptual dysfunction.

\section{ACKNOWLEDGMENTS}

We would like to express our sincere gratitude to Mr. Tatsuya Abe, graduate school of engineering, Hiroshima University, for his helping the experiments. 

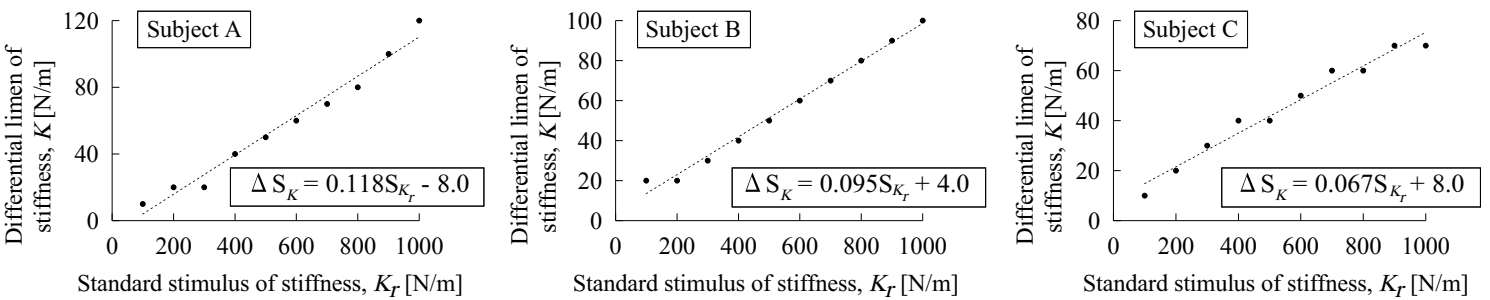

(a) Stiffness
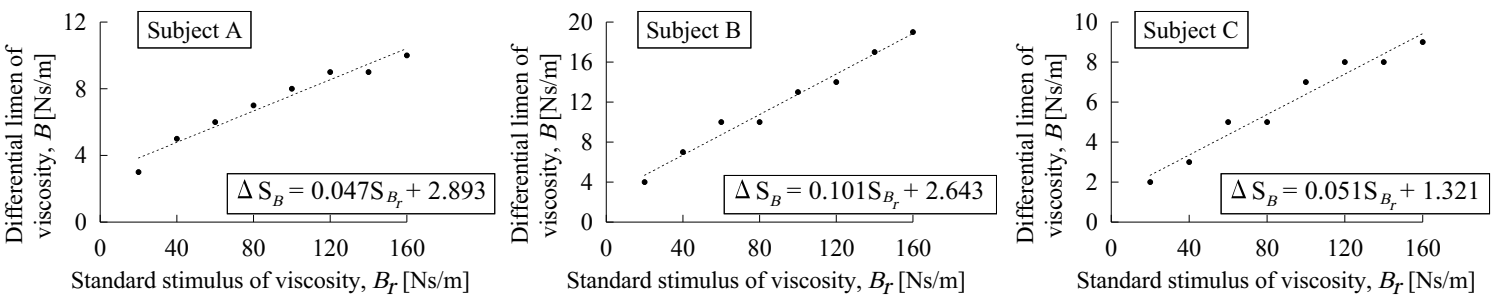

(b) Viscosity
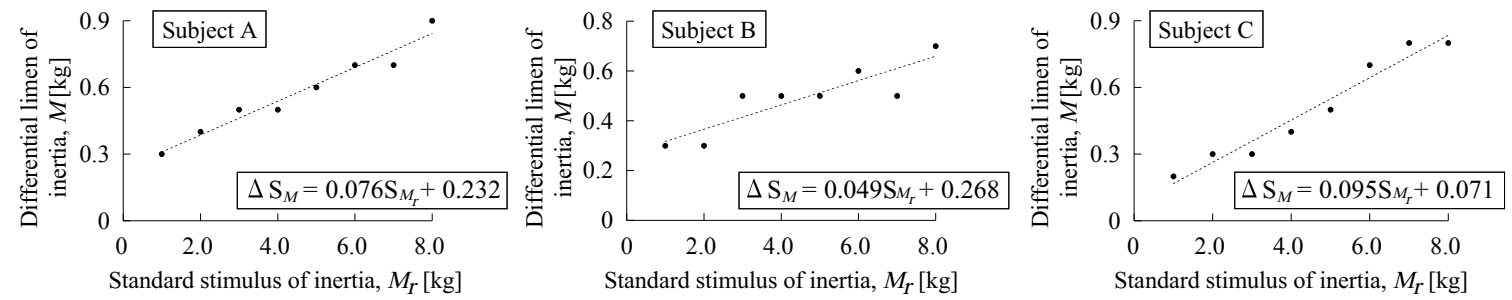

(c) Inertia

Fig. 6. Change of the differential limen for each impedance element by Sub. A, B and C.

\section{References}

Dolan, J.M., Friendman, M.B., and Nagarka, M.L., (1993), Dynamics and loaded impedance components in the maintenance of human arm posture, IEEE Transaction on Systems, Man and Cybernetics, 23, 3, 698-709.

Fujita, K., Sasaki, H., and Koyama, Y., (1999), The experimental examination on hardness sensory mechanism and virtuality stiffness presentation of the human, Society of Biomechanisms 1999 CDROM, 450-455 (in Japanese).

Hogan, N., (1985), Impedance Control: An approach to Manipulation, Parts I, II, III, ASME Journal of Dynamic Systems, Measurement, and Control, 107, 1, 1-24.

Jones, L.A. and Hunter, I.W., (1990), A perceptual analysis of stiffness, Experimental Brain Research, 79, 1, 150-156.

Jones, L.A. and Hunter, I.W., (1993), A perceptual analysis of viscosity," Experimental Brain Research, 94, 2, 343-351.

JUSE the sensory test committee, (1973), Sensory Evaluation Handbook, Union of Japanese Scientists and Engineers.

Kazerooni, H., (1990), Human-Robot Interaction via the Transfer of Power and Information Signals, IEEE Transactions on Systems, Man, and Cybernetics, 20, 2, 450-463.
Kephart, N.C., (1960), The slow learner in the classroom, Charles E. Merril Publishing Co..

Mussa-Ivaldi,F.A., Hogan, N., and Bizzi, E., (1985), Neural, mechanical and geometric factors subserving arm in humans, Journal of Neuroscience, 5, 10, 2732-2743.

Oyama, T., Imai, S., and Wake, T., (1994), Sensory and Perceptual Handbook, Seishin Shobo (in japanese).

Perfetti, C., Miyamoto, S., and Okita, K., (1998), Esercizio Terapeutico Conoscitivio, Kyoudouisyo Syuppansya (in japanese).

Shiomi, K., Kanemitu, Y., and Adachi, A., (1982), Psychological test and measurement Handbook, Nakanishiya Syupan (in Japanese). Srinivasan, M.A. and LaMotte, R.H., (1995), Tactual discrimination of softness, The Journal of Neurophysiology, 73, 1, 88-101.

Tsuji, T., Morasso, P.G., Goto, K., and Ito, K., (1995), Human hand impedance characteristics during maintained posture, Biological Cybernetics, 72, 457-485.

Tsuji, T., Kanji, Y., Kato, T., Kaneko, M., and Kawamura, S., (1999), Impedance Training: Can We Regulate Our Hand Impedance Through Training?, Transaction of the Society of Instrument and Control Engineers, 35, 10, 1300-1306 (in Japanese). 